\title{
The Effect of Intravenous Dexamethasone and Lidocaine on Propofol-Induced Vascular Pain: A Randomized Double-Blinded Placebo-Controlled Trial
}

\author{
Shireen Ahmad, Gildasio S. De Oliveira Jr., Paul C. Fitzgerald, and Robert J. McCarthy \\ Department of Anesthesiology, Northwestern University Feinberg School of Medicine, 251 East Huron Street, Suite F5-704, \\ Chicago, IL 60611, USA \\ Correspondence should be addressed to Gildasio S. De Oliveira Jr.; g-jr@northwestern.edu
}

Received 8 May 2013; Revised 25 June 2013; Accepted 27 June 2013

Academic Editor: Pierangelo Geppetti

Copyright (C) 2013 Shireen Ahmad et al. This is an open access article distributed under the Creative Commons Attribution License, which permits unrestricted use, distribution, and reproduction in any medium, provided the original work is properly cited.

\begin{abstract}
Background. The mechanism for pain associated with intravenous administration of propofol is believed to be related to the release of nitric oxide. We hypothesized that pain following propofol injection would be reduced by pretreatment with dexamethasone. Methods. One hundred fourteen female subjects received $5 \mathrm{~mL}$ of preservative-free saline, $0.5 \mathrm{mg} \cdot \mathrm{kg}^{-1}$ of lignocaine hydrochloride $10 \mathrm{mg} \cdot \mathrm{mL}^{-1}$ or $0.25 \mathrm{mg} \cdot \mathrm{kg}^{-1}$ of dexamethasone, intravenously, following exsanguination and occlusion of the veins of the arm. This was followed by a $0.5 \mathrm{mg} \cdot \mathrm{kg}^{-1}$ injection of propofol. Pain scores, facial grimacing, arm withdrawal, and vocalization were recorded prior to and at 15 and 30 seconds following the injection of propofol. Results. The incidence of moderate to severe pain following the injection of propofol was significantly decreased with both lidocaine and dexamethasone. Hand withdrawal was also significantly decreased in comparison to saline. Conclusion. Low dose dexamethasone is commonly used as an antiemetic, and, in larger doses, it has been demonstrated to provide prolonged postoperative analgesia. At higher analgesic doses, dexamethasone may also reduce pain associated with the injection of propofol. This effect is probably related to the effect of the steroid on nitric oxide production associated with intravenous propofol injection.
\end{abstract}

\section{Introduction}

Propofol (Diprivan, 2,6-di-isopropylphenol) was introduced into clinical use in 1986 and has now become the most widely used intravenous anaesthetic, despite the high incidence of localized pain on injection. In studies of propofol injection into an intravenous catheter in a forearm cephalic vein or vein on the back of the hand, the incidence of pain was $68 \%-72 \%[1,2]$. The pain is immediate and can be profound, and it has been ranked the seventh out of the 33 anesthesia outcomes deserving high priority for improvement, by a panel of anaesthesiologists from academic and community practices [3]. In addition, the hyperdynamic cardiovascular response to the pain can precipitate adverse events in highrisk patients with history of coronary artery disease and/or abnormal heart rhythm.

Clinical strategies designed to alleviate propofol-related pain have been described in the literature including cooling the extremity, dilution of the propofol solution injecting propofol into a large antecubital vein, and the application of topical nitroglycerin on the skin overlying the tip of the intravenous catheter. Injection of lidocaine to prevent propofol injection pain is the most extensively studied technique and is commonly used in clinical practice but with variable results [4]. Our group has recently demonstrated that dexamethasone is an effective strategy to minimize postoperative pain and PONV $[5,6]$. In addition, the administration of intravenous dexamethasone has not been associated with increased incidence of infection or altered hyperglycemic response in the perioperative period $[7,8]$. However, it remains to be determined whether the preoperative administration of intravenous dexamethasone can also reduce the incidence of pain on injection of propofol, which would justify the administration of the drug before anesthetic induction.

Propofol has been shown to release nitric oxide (NO) from vessels in animals and humans, and the release of nitric oxide has been linked to the generation of pain in 
the veins in humans [9-11]. The effects of corticosteroids such as dexamethasone on NO production have been previously demonstrated [12, 13]. In addition, the efficacy of steroids to alter nitric oxide release has also been demonstrated in several disease conditions $[14,15]$. Therefore, the choice of dexamethasone to minimize propofol-induced vascular pain was not only based on its wide clinical utilization but also due to its biological basis.

The purpose of this study was to compare pain scores and behavioral signs of discomfort among groups pretreated with high-dose dexamethasone, lidocaine, or saline that received a propofol bolus injection. We hypothesize that subjects receiving dexamethasone would have less discomfort than the ones receiving saline.

\section{Materials and Methods}

Following the approval by the Institutional Review Board of Northwestern University, informed consent was obtained from adult subjects who were older than 18 years, ASA PS I \& II, and scheduled to undergo outpatient gynaecologic surgery under general anaesthesia. Subjects were excluded if they had a hypersensitivity to propofol or soy bean oil, glycerol, egg lecithin, or sodium oleate, if they had small caliber veins on the dorsum of the hands, if they required intravenous drug administration prior to induction of anaesthesia, or if they required a rapid sequence induction of anaesthesia. Pregnant or lactating patients and those with a history of chronic pain, with neurologic, psychiatric, significant cardiac, renal, or liver disease, or taking sedatives or analgesics preoperatively were excluded.

Subjects were randomly assigned (computer-generated table) to one of the following three pretreatment groups: preservative-free, saline, $0.5 \mathrm{mg} \cdot \mathrm{kg}^{-1}$ of lignocaine hydrochloride $10 \mathrm{mg} \cdot \mathrm{mL}^{-1}$ or $0.25 \mathrm{mg} \cdot \mathrm{kg}^{-1}$ of dexamethasone sodium phosphate. The study drug was diluted with preservative-free saline to a final volume of $5 \mathrm{~mL}$. Prior to the transference to the operating room, an 18-gauge intravenous cannula was inserted into the largest vein on the dorsum of the nondominant hand, and an infusion of Lactated Ringer's solution was started at a rate of $5 \mathrm{~mL} \cdot \mathrm{kg} \cdot \mathrm{hr}^{-1}$. In addition, an appropriately sized blood pressure cuff was placed on the upper arm above the intravenous site. Subjects were asked to rate the severity of pain experienced on insertion of the IV cannula using a visual analogue scale (VAS) and a verbal rating score (VRS) for pain $(0=$ no pain, $1=$ mild pain, $2=$ moderate pain, and $3=$ severe pain). No other drugs were administered through the IV cannula prior to the administration of the study drugs. The infusion was stopped, and the arm was elevated for 15 seconds. The blood pressure cuff was inflated by activating the "Start venous stasis" button on the physiological monitor (Datex-Engstrom, Helsinki, Finland), and the pretreatment study drug was injected $(5 \mathrm{~s})$ into the injection port closest to the cannulation site. The study medication was prepared by a single investigator (Paul C. Fitzgerald), and the investigator who administered the study drugs was blind to the study group. The patient was then asked to rate the discomfort associated with the injection using the VRS scale. Behavioral signs (facial grimacing, arm withdrawal, and vocal response) were also recorded following the injection.

Two minutes following the study drug administration, the blood pressure was deflated by activating the "Stop venous stasis" button, and the intravenous infusion was restarted by releasing the roller clamp. The $0.5 \mathrm{mg} \cdot \mathrm{kg}^{-1}$ propofol was injected through the same injection port over 5 seconds. Spontaneous complaints of pain were noted. If there were none, 15 and 30 seconds following the injection the patient was asked to rate the discomfort (VRS) associated with the propofol injection. Behavioral signs were also recorded. After the last recording, anaesthesia was induced in the standard manner, and anesthetic management was at the discretion of the anaesthesiologist. Subjects were contacted $24 \mathrm{~h}$ following surgery and questioned about pain or swelling at the injection site.

\section{Statistical Analysis}

The primary outcome variable was the incidence of moderate to severe pain reported. Based on the review of the literature, we anticipated that 65 percent of those treated with saline, 30 percent of those treated with lignocaine, and 40 percent of those treated with dexamethasone would report moderate to severe pain. Assuming these rates of occurrence, an effect size $(W)$ of 0.29 was calculated. A sample size of 115 achieves $80 \%$ power to detect a significant difference among groups at "alpha" of 0.05 using the two-degree freedom $\chi^{2}$ test. Clinical characteristics among the groups were compared using oneway ANOVA or the Kruskal-Wallis $H$ test. The incidence of moderate/severe pain and the number of patients reporting pain following propofol injection, the incidence of behavioral signs, and other adverse effects were compared using a chisquare analysis. Estimates of exact $P$ values were determined for the Mann-Whitney and the $\chi^{2}$ test using the Monte Carlo method with 10,000 samples and confidence limits of $99 \%$. All tests are reported as two sided, and a $P<0.05$ was required to reject the null hypothesis. Nominal and categorical data are presented as counts and percentage of respondents. Interval data are presented as medians with interquartile range (IRQ). All reported $P$ values are two tailed. Data were analyzed using NCSS 2007 version 7.1.20, release date 2/19/2010, NCSS LLC, Kaysville, UT, USA, and PASW Statistics 18.0.2, release date 4/2/2010, SPSS, Inc., Chicago, IL, USA.

\section{Results}

One hundred and twenty-two subjects were enrolled. Eight subjects were excluded from the study prior to randomization: 1 due to a changed anaesthetic plan, 4 due to cancellation of the procedure, 2 due to pain prior to the study drug administration at the intravenous site, and 1 because a nonstudy drug was administered. The median age of the subjects was 37 years (interquartile range: 32 to $42 \mathrm{y}$ ), and weight was $65 \mathrm{~kg}$ (interquartile range: 55 to $77 \mathrm{~kg}$ ) and did not differ among groups.

All subjects were awake and alert during the period of pain assessment following injection of propofol. The median 
TABLE 1: Pain and behavioral responses following propofol administration.

\begin{tabular}{|c|c|c|c|c|c|c|c|c|}
\hline & $\begin{array}{l}\text { Saline } \\
(n=37)\end{array}$ & $\begin{array}{l}\text { Lidocaine } \\
(n=43)\end{array}$ & $\begin{array}{l}\text { Dexamethasone } \\
(n=34)\end{array}$ & $P$ & $\begin{array}{l}\text { Saline } \\
(n=37)\end{array}$ & $\begin{array}{l}\text { Lidocaine } \\
(n=43)\end{array}$ & $\begin{array}{l}\text { Dexamethasone } \\
\quad(n=34)\end{array}$ & $P$ \\
\hline Time (s) & & 15 & & & & 30 & & \\
\hline Moderate/severe pain $n(\%)$ & $18(56)$ & $6(14)$ & $8(24)$ & 0.002 & $22(60)$ & $11(26)$ & $14(41)$ & 0.009 \\
\hline \multicolumn{9}{|l|}{ Pain intensity $(n)$} \\
\hline None & 13 & 30 & 22 & \multirow{4}{*}{0.03} & 5 & 24 & 15 & \multirow{4}{*}{0.01} \\
\hline Mild & 6 & 7 & 4 & & 10 & 8 & 5 & \\
\hline Moderate & 7 & 2 & 3 & & 15 & 8 & 10 & \\
\hline Severe & 11 & 4 & 5 & & 7 & 3 & 4 & \\
\hline Facial grimacing $n(\%)$ & 0 & $1(2)$ & $2(6)$ & 0.29 & $5(14)$ & $3(7)$ & $0(0)$ & 0.07 \\
\hline Hand withdrawal $n(\%)$ & $17(46)$ & $3(7)$ & $5(15)$ & $<0.005$ & $21(58)$ & $5(12)$ & $10(29)$ & $<0.005$ \\
\hline Crying $n(\%)$ & $1(3)$ & $0(0)$ & $1(3)$ & 0.53 & $0(0)$ & $1(2)$ & $0(0)$ & 0.44 \\
\hline
\end{tabular}

VAS for reported pain during IV cannula insertion was 2 (interquartile range: 0 to 6.6 ) in the saline group, 5 (1 to 10) in the lignocaine group, and 3 (0 to 13.5) in the dexamethasone group $(P=0.20)$. The mean (SD) doses $(\mathrm{mg})$ of propofol administered to the saline, lignocaine, and dexamethasone groups were the following: $30.8 \pm 3.6,30.2 \pm 2.9$, and $31.2 \pm 3.3$ $(P=0.48)$, respectively.

The incidence of moderate to severe pain at $15 \mathrm{~s}$ after the injection of propofol was $56 \%$ in the saline group, $14 \%$ in the lignocaine group, and $24 \%$ in the dexamethasone group $(P=$ 0.002 ), and the incidence of moderate to severe pain at $30 \mathrm{~s}$ after the injection of propofol was $60 \%$ in the saline group, $26 \%$ in the lignocaine group, and $41 \%$ in the dexamethasone group $(P=0.009)$ (Table 1$)$. At $15 \mathrm{~s}$, dexamethasone reduced the number of subjects reporting moderate to severe pain $(P=0.03)$ as well as exhibiting hand withdrawal $(P=0.005)$ compared to saline. Dexamethasone also reduced the number of subjects who exhibited grimacing $(P=0.02)$ and hand withdrawal $(P=0.02)$ compared to saline at $30 \mathrm{~s}$. Lignocaine and dexamethasone did not differ in the reported pain or behavioral signs at either time.

Twenty-four hours postoperatively, 2 subjects reported mild pain in the study arm (1 saline and 1 lignocaine), and 1 subject (lignocaine) reported swelling at the catheter site. No other complications were noted.

\section{Discussion}

The most important finding of this study was the reduction in the number of subjects that reported moderate or severe pain following propofol administration when pretreated with dexamethasone compared to saline. Systemic dexamethasone has been commonly used perioperatively to minimize postoperative nausea and vomiting and to improve overall quality of recovery $[5,6]$. In addition, dexamethasone has been shown to decrease nitric oxide production which has been shown to mediate propofol-induced vascular pain [1113]. The current study suggests that the preoperative administration of dexamethasone also diminishes pain on propofol injection.
The need to treat propofol-induced nociception is essential not only because it is unpleasant, but also because it can lead to serious sequelae such as myocardial ischemia when hemodynamic changes occur in response to the pain associated with injection [16]. The reduction in propofolinduced pain behaviors was also achieved with lidocaine in the current investigation. Nevertheless, dexamethasone has an advantage over lidocaine to improve postoperative quality of recovery since it does not require the additional administration of an intraoperative infusion [17].

Studies suggest that the mechanisms underlying nociception from vascular tissues following propofol injection are multifactorial in origin. Propofol has been demonstrated in vitro to stimulate nitric oxide (NO) release [18]. Nociceptive nerve endings have been found in the endothelium of veins in humans, a well-known source of NO, suggesting a role of NO in nociception $[19,20]$. In addition, NO from the vascular endothelium binds to guanyl cyclase which catalyzes the conversion of guanosine triphosphate to guanosine monophosphate, which facilitates PGE2-induced hyperalgesia [11]. It has been found that pain following intravenous injection of bradykinin and hyperosmolar solutions can be blocked by pretreatment with NO synthase (NOS) inhibitor, suggesting that an intact NOS pathway is needed to elicit vascular nociception [21].

Previous investigators have described various attempts to eliminate propofol-induced vascular pain [22]. Many of these studies lack a scientific rationale, and the pharmacologic interventions often tested do not have a biologic basis. The effects of anesthetics and perioperative stress on NO production have been previously demonstrated $[23,24]$. In patients with asthma, corticosteroids reduced the levels of exhaled NO, and dexamethasone inhibited nitrite production in cells from the human joint and lung epithelial cells [12]. Dexamethasone is commonly used perioperatively as an antiemetic in both adults and children in doses ranging from $150 \mu \mathrm{g} / \mathrm{kg}$ to $0.5 \mathrm{mg} / \mathrm{kg}[25,26]$.

There are limitations to our study design that may have affected our findings. We used a single dose of dexamethasone $(0.25 \mathrm{mg} / \mathrm{kg})$ which is greater than the dose commonly used as an antiemetic, but it is comparable to doses that have 
been shown to provide a prolonged postoperative analgesic effect without adverse effects. Furthermore, it has been noted that patients receiving dexamethasone $20 \mathrm{mg}$ daily for five days to control chemotherapy-induced nausea and vomiting had no evidence of immunosuppression or hypothalamicpituitary-adrenal axis dysfunction [27]. A limitation of all studies evaluating vascular pain following propofol administration is the use of a subhypnotic dose of propofol so that reliable pain assessments reporting can be obtained. Finally, our study was underpowered to detect the difference in the incidence of moderate to severe pain between lidocaine and dexamethasone.

In conclusion, the findings of this study suggest the effectiveness of dexamethasone pretreatment in reducing vascular pain following propofol administration. Pretreatment with dexamethasone was more effective than saline and had a similar efficacy as lidocaine prior to propofol injection. Since dexamethasone is commonly used to prevent postoperative nausea and vomiting and to improve post-surgical recovery, clinical practitioners should consider using dexamethasone preoperatively to minimize propofol-induced vascular pain and its undesirable side effects.

\section{Conflict of Interests}

The authors have no conflict of interests to disclose.

\section{Funding}

Financial support was provided by departmental funds.

\section{References}

[1] C.-L. Huang, Y.-P. Wang, Y.-J. Cheng, L. Susetio, and C.-C. Liu, "The effect of carrier intravenous fluid speed on the injection pain of propofol," Anesthesia and Analgesia, vol. 81, no. 5, pp. 1087-1088, 1995.

[2] G. Gehan, P. Karoubi, F. Quinet, A. Leroy, C. Rathat, and J. L. Pourriat, "Optimal dose of lignocaine for preventing pain on injection of propofol," British Journal of Anaesthesia, vol. 66, no. 3, pp. 324-326, 1991.

[3] A. Macario, M. Weinger, P. Truong, and M. Lee, "Which clinical anesthesia outcomes are both common and important to avoid? The perspective of a panel of expert anesthesiologists," Anesthesia and Analgesia, vol. 88, no. 5, pp. 1085-1091, 1999.

[4] P. Picard and M. R. Tramèr, "Prevention of pain on injection with propofol: a quantitative systematic review," Anesthesia and Analgesia, vol. 90, no. 4, pp. 963-969, 2000.

[5] G. S. De Oliveira Jr., L. J. Castro-Alves, S. Ahmad, M. C. Kendall, and R. J. McCarthy, "Dexamethasone to prevent postoperative nausea and vomiting: an updated meta-analysis of randomized," Anesthesia \& Analgesia, vol. 116, pp. 58-74, 2013.

[6] G. S. De Oliveira Jr., S. Ahmad, P. C. Fitzgerald et al., "Dose ranging study on the effect of preoperative dexamethasone on postoperative quality of recovery and opioid consumption after ambulatory gynaecological surgery," British Journal of Anaesthesia, vol. 107, no. 3, pp. 362-371, 2011.

[7] C. S. Bolac, A. H. Wallace, G. Broadwater, L. J. Havrilesky, and A. S. Habib, "The impact of postoperative nausea and vomiting prophylaxis with dexamethasone on postoperative wound complications in patients undergoing laparotomy for endometrial cancer," Anesthesia \& Analgesia, vol. 116, pp. 10411047, 2013.

[8] B. B. Abdelmalak, A. M. Bonilla, D. Yang et al., "The hyperglycemic response to major noncardiac surgery and the added effect of steroid administration in patients with and without diabetes," Anesthesia \& Analgesia, vol. 116, pp. 1116-1122, 2013.

[9] W. K. Park, C. Lynch III, and R. A. Johns, "Effects of propofol and thiopental in isolated rat aorta and pulmonary artery," Anesthesiology, vol. 77, no. 5, pp. 956-963, 1992.

[10] L. Moreno, M. A. Martínez-Cuesta, V. Muedra, B. Beltrán, and J. Esplugues, "Role of the endothelium in the relaxation induced by propofol and thiopental in isolated arteries from man," Journal of Pharmacy and Pharmacology, vol. 49, no. 4, pp. 430-432, 1997.

[11] D. Kindgen-Milles and J. O. Arndt, "Nitric oxide as a chemical link in the generation of pain from veins in humans," Pain, vol. 64, no. 1, pp. 139-142, 1996.

[12] S. A. Kharitonov, D. H. Yates, and P. J. Barnes, "Inhaled glucocorticoids decrease nitric oxide in exhaled air of asthmatic patients," American Journal of Respiratory and Critical Care Medicine, vol. 153, no. 1, pp. 454-457, 1996.

[13] P. S. Grabowski, H. Macpherson, and S. H. Ralston, "Nitric oxide production in cells derived from the human joint," British Journal of Rheumatology, vol. 35, no. 3, pp. 207-212, 1996.

[14] Y. Huo, P. Rangarajan, E.-A. Ling, and S. T. Dheen, "Dexamethasone inhibits the Nox-dependent ROS production via suppression of MKP-1-dependent MAPK pathways in activated microglia," BMC Neuroscience, vol. 12, article 49, 2011.

[15] R. Aras-López, F. E. Xavier, M. Ferrer, and G. Balfagón, "Dexamethasone decreases neuronal nitric oxide release in mesenteric arteries from hypertensive rats through decreased protein kinase C activation," Clinical Science, vol. 117, no. 8, pp. 305-312, 2009.

[16] T. Morishima, K. Sobue, H. Arima et al., "Profound pain due to propofol injection triggered myocardial ischemia in a patient with a suspected pheochromocytoma," Anesthesia \& Analgesia, vol. 96, no. 2, p. 631, 2003.

[17] G. S. De Oliveira Jr., P. Fitzgerald, L. F. Streicher, R. J. Marcus, and R. J. McCarthy, "Systemic lidocaine to improve postoperative quality of recovery after ambulatory laparoscopic surgery," Anesthesia \& Analgesia, vol. 115, pp. 262-267, 2012.

[18] A. J. Petros, R. G. Bogle, and J. D. Pearson, "Propofol stimulates nitric oxide release from cultured porcine aortic endothelial cells," British Journal of Pharmacology, vol. 109, no. 1, pp. 6-7, 1993.

[19] F. S. Gragasin, S. L. Bourque, and S. T. Davidge, "Propofol increases vascular relaxation in aging rats chronically treated with the angiotensin-converting enzyme inhibitor captopril," Anesthesia \& Analgesia, vol. 116, pp. 775-783, 2013.

[20] T. R. L. Romero, G. S. Galdino, G. C. Silva et al., "Ketamine activates the 1-arginine/nitric oxide/cyclic guanosine monophosphate pathway to induce peripheral antinociception in rats," Anesthesia and Analgesia, vol. 113, no. 5, pp. 1254-1259, 2011.

[21] M. Nakane and H. Iwama, "A potential mechanism of propofolinduced pain on injection based on studies using nafamostat mesilate," British Journal of Anaesthesia, vol. 83, no. 3, pp. 397404, 1999.

[22] T. Shimizu, S. Inomata, and M. Tanaka, "Rapid injection of propofol reduces vascular pain and facilitates Laryngeal Mask Airway insertion," Journal of Clinical Anesthesia, vol. 23, no. 7, pp. 540-543, 2011. 
[23] X. Feng, J. J. Liu, X. Zhou et al., "Single sevoflurane exposure decreases neuronal nitric oxide synthase levels in the hippocampus of developing rats," British Journal of Anaesthesia, vol. 109, pp. 225-233, 2012.

[24] K. Hama-Tomioka, H. Kinoshita, K. Nakahata et al., "Roles of neuronal nitric oxide synthase, oxidative stress, and propofol in N-methyl-D-aspartate-induced dilatation of cerebral arterioles," British Journal of Anaesthesia, vol. 108, no. 1, pp. 21-29, 2012.

[25] R. Holt, P. Rask, K. P. Coulthard et al., "Tropisetron plus dexamethasone is more effective than tropisetron alone for the prevention of postoperative nausea and vomiting in children undergoing tonsillectomy," Paediatric Anaesthesia, vol. 10, no. 2, pp. 181-188, 2000.

[26] J.-J. Wang, S.-T. Ho, J.-I. Tzeng, and C.-S. Tang, "The effect of timing of dexamethasone administration on its efficacy as a prophylactic antiemetic for postoperative nausea and vomiting," Anesthesia and Analgesia, vol. 91, no. 1, pp. 136-139, 2000.

[27] G. Del Priore, K. J. Gurski, D. P. Warshal, C. Angel, and B. Dubeshter, "Adrenal function following high-dose steroids in ovarian cancer patients," Gynecologic Oncology, vol. 59, no. 1, pp. 102-104, 1995. 


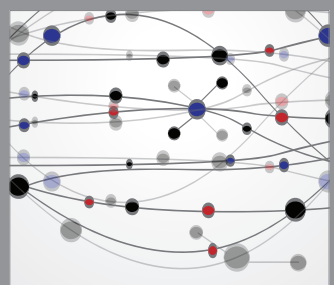

The Scientific World Journal
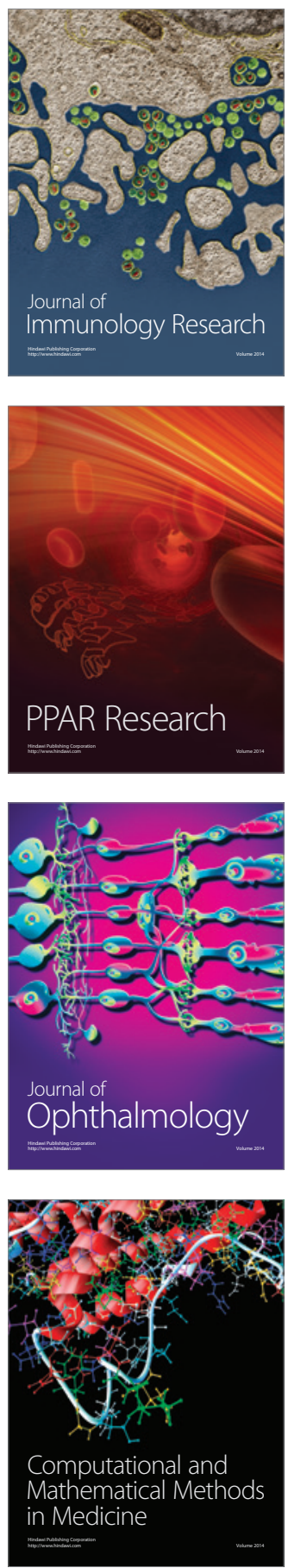

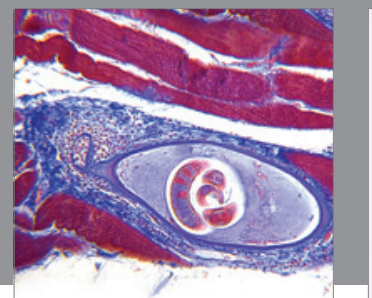

Gastroenterology

Research and Practice
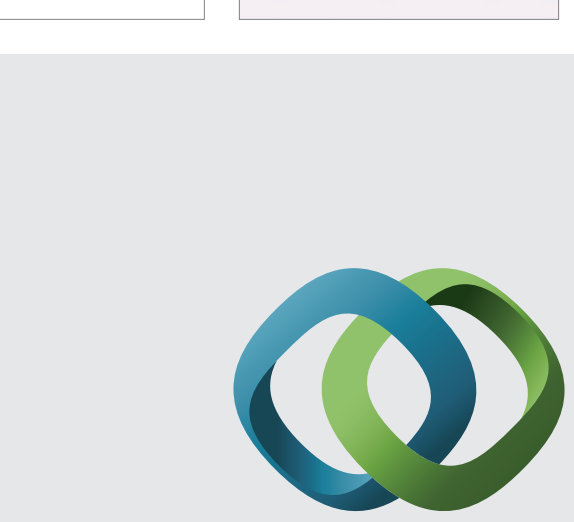

\section{Hindawi}

Submit your manuscripts at

http://www.hindawi.com
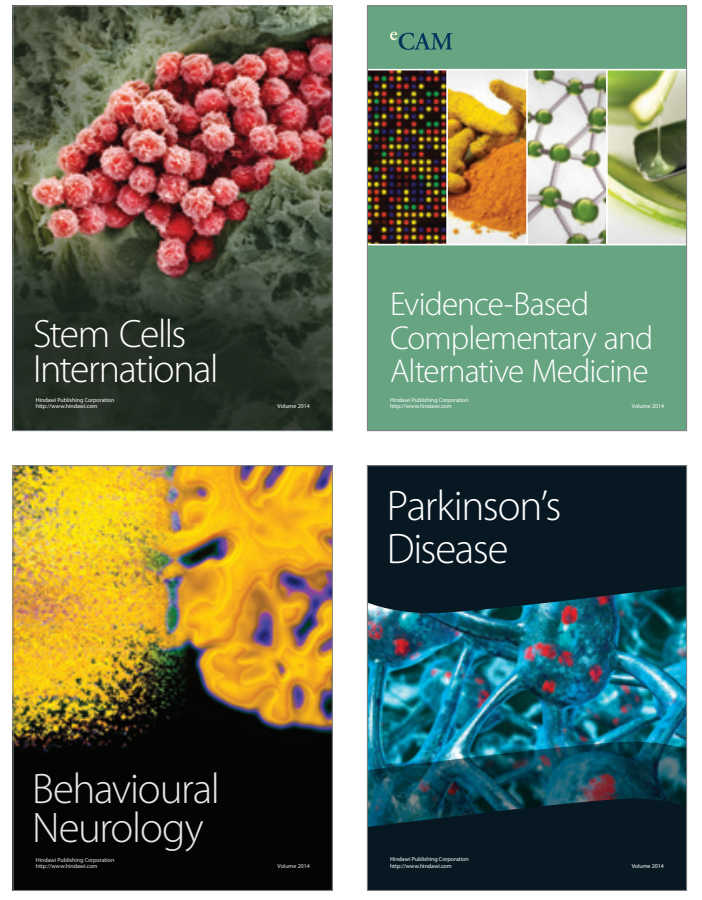
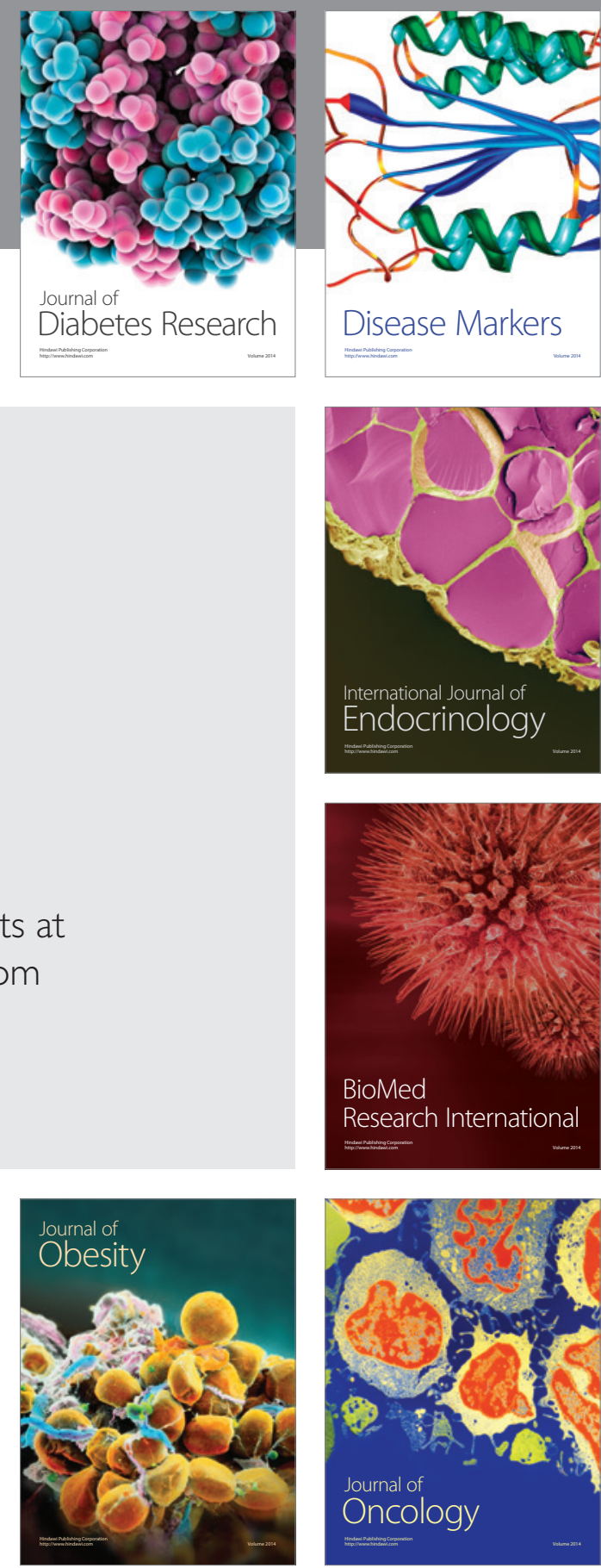

Disease Markers
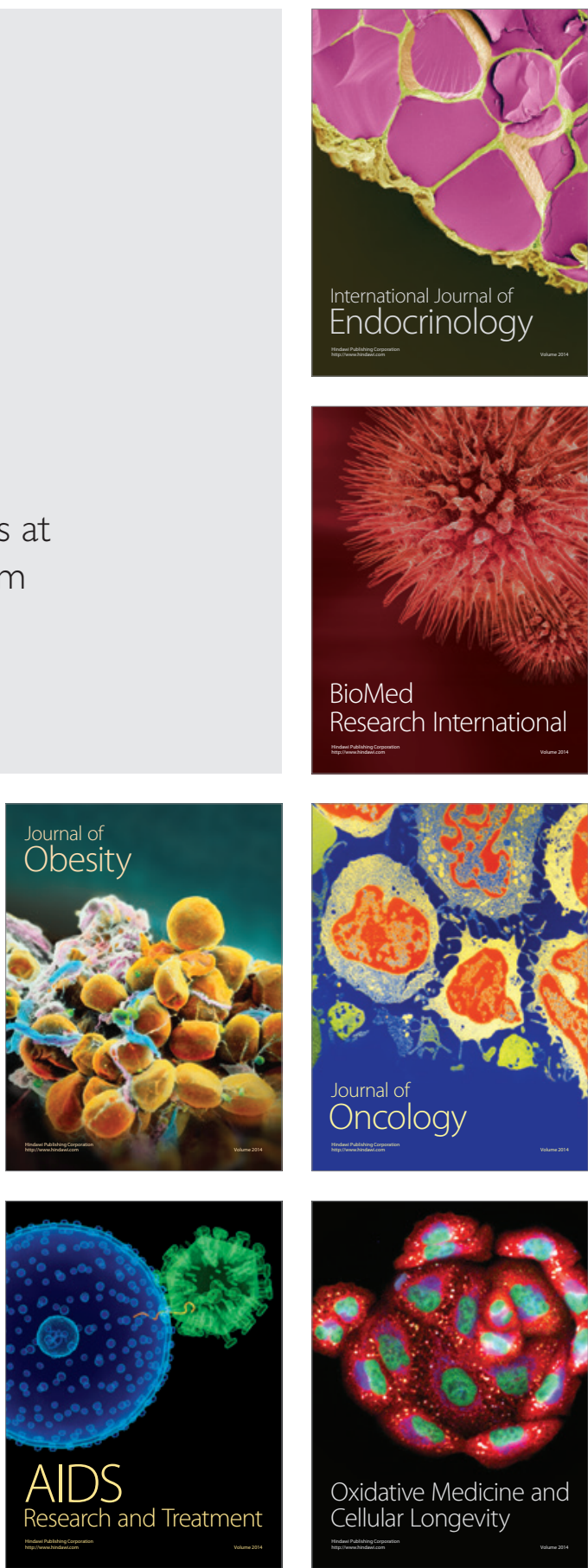